Bull. Korean Math. Soc. 48 (2011), No. 6, pp. 1219-1224

http://dx.doi.org/10.4134/BKMS.2011.48.6.1219

\title{
A DETERMINANT FORMULA FOR CONGRUENT ZETA FUNCTIONS OF REAL ABELIAN FUNCTION FIELDS
}

\author{
JAEHYun AhN AND Hwanyup Jung
}

\begin{abstract}
In this paper we give a determinant formula for congruent zeta functions of real Abelian function fields. We also give some examples of congruent zeta functions when the conductor of real Abelian function field is monic irreducible.
\end{abstract}

\section{Introduction}

Let $\mathrm{k}=\mathbb{F}_{q}(T)$ be the rational function field over the finite field $\mathbb{F}_{q}$ and $\mathbb{A}=\mathbb{F}_{q}[T]$. Let $\infty$ be the place of $\mathrm{k}$ associated to $1 / T$, which is called the infinite one of $\mathrm{k}$. Write $\mathbb{A}^{+}=\{1 \neq M \in \mathbb{A}: M$ is monic $\}$ and $\mathbb{A}_{\text {irr }}^{+}=\{P \in$ $\mathbb{A}^{+}: P$ is irreducible $\}$. For any $M \in \mathbb{A}^{+}$, write $K_{M}$ for the $M$ th cyclotomic function field and $K_{M}^{+}$for the maximal real subfield of $K_{M}$. In this paper, by an Abelian function field, we always mean a finite Abelian extension $F$ of k which is contained in a cyclotyomic function field $K_{M}$, and $F$ is said to be real if $\infty$ splits completely in $F$. Let $N=N(F) \in \mathbb{A}^{+}$be the conductor of $F$, that is, $K_{N}$ is the smallest cyclotomic function field containing $F$. For such a field $F$, there exists a polynomial $P_{F}(X) \in \mathbb{Z}[X]$ such that

$$
\zeta(s, F)=\frac{P_{F}\left(q^{-s}\right)}{\left(1-q^{-s}\right)\left(1-q^{1-s}\right)},
$$

where $\zeta(s, F)$ is the congruence zeta function of $F$, and $P_{F}(1)$ is equal to the divisor class number $h_{F}$ of $F$. In recent paper [5], Shiomi has expressed the polynomial $P_{K_{M}^{+}}(X)$ as determinant of matrix $D_{K_{M}^{+}}(X)$ with entries in $\mathbb{Z}[X]$ up to some polynomial $J_{K_{M}^{+}}(X)$. Since $h_{K_{M}^{+}}=P_{K_{M}^{+}}(1)$, this determinant formula for $P_{K_{M}^{+}}(X)$ can be regarded as generalization of that for class number $h_{K_{M}}^{+}$ $([1],[3])$.

Received July 7, 2010

2010 Mathematics Subject Classification. 11R58, 11R60, $11 \mathrm{M} 38$.

Key words and phrases. congruent zeta function, Abelian function field.

This research was supported by Basic Science Research Program through the National Research Foundation of Korea(NRF) funded by the Ministry of Education, Science and Technology(2011-0005138). 
The aim of this paper is to give a determinant formula for the polynomial $P_{F}(X)$ for arbitrary real Abelian function field $F$. We also give some examples of the polynomials $P_{F}(X)$ when $F$ is a real Abelian function field of monic irreducible conductor and $q=3$.

\section{Zeta and $L$-functions}

Let $\zeta(s, F)$ be the congruence zeta function of a real Abelian function field $F$ given by

$$
\zeta(s, F)=\prod_{\mathfrak{p}}\left(1-\frac{1}{N \mathfrak{p}^{s}}\right)^{-1},
$$

where $\mathfrak{p}$ runs over all primes of $F$. It is well known that there exists a polynomial $P_{F}(X) \in \mathbb{Z}[X]$ of degree $2 g$, where $g$ is the genus of $F$, such that

$$
\zeta(s, F)=\frac{P_{F}\left(q^{-s}\right)}{\left(1-q^{-s}\right)\left(1-q^{1-s}\right)} .
$$

Moreover, the polynomial $P_{F}(X)$ satisfies $P_{F}(0)=1$ and $P_{F}(1)=h_{F}$, where $h_{F}$ is the divisor class number of $F$. Let $\mathcal{O}_{F}$ be the integral closure of $\mathbb{A}$ in $F$ and $\zeta\left(s, \mathcal{O}_{F}\right)$ be the zeta function of $\mathcal{O}_{F}$ given by

$$
\zeta\left(s, \mathcal{O}_{F}\right)=\prod_{\mathfrak{p}}\left(1-\frac{1}{N \mathfrak{p}^{s}}\right)^{-1},
$$

where $\mathfrak{p}$ runs over all prime ideals of $\mathcal{O}_{F}$. Since $\infty$ splits completely in $F$, the functions $\zeta(s, F)$ and $\zeta\left(s, \mathcal{O}_{F}\right)$ satisfy the following equality

$$
\zeta(s, F)=\zeta\left(s, \mathcal{O}_{F}\right)\left(1-q^{-s}\right)^{-[F: \mathrm{k}]} .
$$

Let $X_{F}$ be the group of primitive Dirichlet characters of $\mathbb{A}$ associated to $F$. For $\chi \in X_{F}$, let $L(s, \chi)$ be the $L$-function associated to $\chi$ given by

$$
L(s, \chi)=\prod_{P \in \mathbb{A}_{\mathrm{irr}}^{+}}\left(1-\chi(P) q^{-s \operatorname{deg} P}\right)^{-1} .
$$

Then we have

$$
\zeta\left(s, \mathcal{O}_{F}\right)=\prod_{\chi \in X_{F}} L(s, \chi)
$$

Let $\chi_{0} \in X_{F}$ denote the trivial character. Since $L\left(s, \chi_{0}\right)=\left(1-q^{1-s}\right)^{-1}$, from (2.1), (2.2) and (2.3), we get

$$
\prod_{\chi_{0} \neq \chi \in X_{F}} L(s, \chi)=\left(1-q^{-s}\right)^{[F: \mathrm{k}]-1} P_{F}\left(q^{-s}\right) .
$$

For any $\chi \in X_{F}$, let $F_{\chi} \in \mathbb{A}^{+}$be the conductor of $\chi$ and $\tilde{\chi}=\chi \circ \pi_{\chi}$, where $\pi_{\chi}:(\mathbb{A} / N \mathbb{A})^{*} \rightarrow\left(\mathbb{A} / F_{\chi} \mathbb{A}\right)^{*}$ is the canonical homomorphism. Then we have

$$
L(s, \tilde{\chi})=L(s, \chi) \prod_{Q \in \mathbb{A}_{\mathrm{irr}}^{+}, Q \mid N}\left(1-\chi(Q) q^{-s \operatorname{deg} Q}\right) .
$$


Thus, by (2.4) and (2.5), we have

$$
\prod_{\chi_{0} \neq \chi \in X_{F}} L(s, \tilde{\chi})=\left(1-q^{-s}\right)^{[F: \mathrm{k}]-1} P_{F}\left(q^{-s}\right) J_{F}\left(q^{-s}\right),
$$

where $J_{F}(X)$ is the polynomial given by

$$
J_{F}(X)=\prod_{\chi_{0} \neq \chi \in X_{F}} \prod_{Q \in \mathbb{A}_{\mathrm{irr}}^{+}, Q \mid N}\left(1-\chi(Q) X^{\operatorname{deg} Q}\right) .
$$

Finally we give some remarks on the polynomial $J_{F}(X)$. They satisfy the following equality (cf. [5, Proposition 3.1])

$$
J_{F}(X)=\prod_{Q \in \mathbb{A}_{\mathrm{irr}}^{+}, Q \mid N} \frac{\left(1-X^{f_{Q} \operatorname{deg} Q}\right)^{g_{Q}}}{1-X^{\operatorname{deg} Q}},
$$

where $f_{Q}$ is the residue class degree of $Q$ in $F / \mathrm{k}$ and $g_{Q}$ is the number of primes over $Q$ in $F$. Hence we see that $J_{F}(X) \in \mathbb{Z}[X]$ and in particular, $J_{F}(X)=1$ if $N$ is a power of some $Q \in \mathbb{A}_{\text {irr }}^{+}$.

\section{A determinant formula for $P_{F}(X)$}

Let $F$ be a real Abelian function field with conductor $N$. Let

$$
\mathcal{R}_{N}=(\mathbb{A} / N \mathbb{A})^{*} / \mathbb{F}_{q}^{*} .
$$

For $\alpha \in(\mathbb{A} / N \mathbb{A})^{*}$, there exists a unique polynomial $A_{\alpha} \in \mathbb{A}$ such that $\operatorname{deg} A_{\alpha}<$ $\operatorname{deg} N$ and $A_{\alpha}+N \mathbb{A}=\alpha$. Write $\operatorname{sgn}_{N}(\alpha) \in \mathbb{F}_{q}^{*}$ for the leading coefficient of $A_{\alpha}, \operatorname{deg}_{N}(\alpha)=\operatorname{deg} A_{\alpha}$ and $c^{\lambda}(\alpha)=\lambda^{-1}\left(\operatorname{sgn}_{N}(\alpha)\right)$ for any character $\lambda$ of $\mathbb{F}_{q}^{*}$. We can easily see that $\operatorname{deg}_{N}$ is a function over $\mathcal{R}_{N}$. Let $\mathcal{H}$ be the subgroup of $\mathcal{R}_{N}$ which is isomorphic to $\operatorname{Gal}\left(K_{N}^{+} / F\right)$ under the canonical isomorphism $\mathcal{R}_{N} \cong \operatorname{Gal}\left(K_{N}^{+} / \mathrm{k}\right)$. For each $\sigma \in \operatorname{Gal}(F / \mathrm{k})$, choose $\beta_{\sigma} \in(\mathbb{A} / N \mathbb{A})^{*}$ which corresponds to $\sigma$ under $(\mathbb{A} / N \mathbb{A})^{*} \rightarrow \mathcal{R}_{N} \rightarrow \mathcal{R}_{N} / \mathcal{H} \cong \operatorname{Gal}(F / \mathrm{k})$. Choose a subset $\Omega_{\mathcal{H}} \subseteq(\mathbb{A} / N \mathbb{A})^{*}$ which are mapped bijectively onto $\mathcal{H}$ under the homomorphism $(\mathbb{A} / N \mathbb{A})^{*} \rightarrow \mathcal{R}_{N}$. For each $\sigma \in \operatorname{Gal}(F / \mathrm{k})$, define a polynomial $f_{\sigma}(X) \in \mathbb{Z}[X]$ by

$$
f_{\sigma}(X):=\sum_{\alpha \in \Omega_{\mathcal{H}}} X^{\operatorname{deg}_{N}\left(\beta_{\sigma} \alpha\right)} .
$$

Since $\left\{\beta_{\sigma} \alpha: \sigma \in \operatorname{Gal}(F / \mathrm{k}), \alpha \in \Omega_{\mathcal{H}}\right\}$ forms a complete system of representatives of $\mathcal{R}_{N}$, we can see easily that $f_{\sigma}(X)$ is independent of the choices of $\Omega_{\mathcal{H}}$ and $\beta_{\sigma}$. Define a matrix $D_{F}(X)$ by

$$
D_{F}(X):=\left(\frac{f_{\sigma \tau^{-1}}(X)-f_{\sigma}(X)}{1-X}\right)_{1 \neq \sigma, \tau \in \operatorname{Gal}\left(F^{+} / \mathrm{k}\right)} .
$$

Theorem 3.1. With notations as above, we have

$$
\operatorname{det} D_{F}(X)=P_{F}(X) J_{F}(X) \text {. }
$$


Proof. For $\chi_{0} \neq \chi \in X_{F}$, as in the proof of [5, Theorem 3.1], we have

$$
\begin{aligned}
L(s, \tilde{\chi}) & =\sum_{\sigma \in \operatorname{Gal}(F / \mathrm{k})} \sum_{\alpha \in \Omega_{\mathcal{H}}} \tilde{\chi}\left(\beta_{\sigma} \alpha\right) q^{-s \operatorname{deg}_{N}\left(\beta_{\sigma} \alpha\right)} \\
& =\sum_{\sigma \in \operatorname{Gal}(F / \mathrm{k})} \tilde{\chi}\left(\beta_{\sigma}\right)\left(\sum_{\alpha \in \Omega_{\mathcal{H}}} q^{-s \operatorname{deg}_{N}\left(\beta_{\sigma} \alpha\right)}\right)=\sum_{\sigma \in \operatorname{Gal}(F / \mathrm{k})} \tilde{\chi}\left(\beta_{\sigma}\right) f_{\sigma}\left(q^{-s}\right) .
\end{aligned}
$$

Thus, by the Frobenius determinant formula,

$$
\begin{aligned}
\prod_{\chi_{0} \neq \chi \in X_{F}} L(s, \tilde{\chi}) & =\prod_{\chi_{0} \neq \chi \in X_{F}} \sum_{\sigma \in \operatorname{Gal}(F / \mathrm{k})} \tilde{\chi}\left(\beta_{\sigma}\right) f_{\sigma}\left(q^{-s}\right) \\
& =\operatorname{det}\left(f_{\sigma \tau^{-1}}\left(q^{-s}\right)-f_{\sigma}\left(q^{-s}\right)\right)_{1 \neq \sigma, \tau \in \operatorname{Gal}(F / \mathrm{k})} .
\end{aligned}
$$

So, by (2.6), we get

$$
\operatorname{det} D_{F}\left(q^{-s}\right)=P_{F}\left(q^{-s}\right) \cdot J_{F}\left(q^{-s}\right) .
$$

Putting $X=q^{-s}$, we get the desired result.

Now we assume that $F$ has a monic irreducible conductor $P \in \mathbb{A}_{i r r}^{+}$of degree $d$. Fix a primitive root $Q$ of $P$ with $\operatorname{deg} Q<d$. For each integer $i \geq 0$, let $Q_{i}$ be the unique polynomial such that $Q^{i} \equiv Q_{i} \bmod P$ and $\operatorname{deg} Q_{i}<d$. Let $r=\frac{q^{d}-1}{q-1}$ and $n=[F: \mathrm{k}]$. Under the isomorphism $(\mathbb{A} / P \mathbb{A})^{*} \rightarrow \operatorname{Gal}\left(K_{P} / \mathrm{k}\right), A+P \mathbb{A} \mapsto$ $\sigma_{A}$, we have $\operatorname{Gal}\left(K_{P} / K_{P}^{+}\right)=\left\{\sigma_{Q_{r i}}: 0 \leq i<q-1\right\}$ and $\operatorname{Gal}\left(K_{P} / F\right)=\left\{\sigma_{Q_{n i}}\right.$ : $\left.0 \leq i<\frac{q^{d}-1}{n}\right\}$. Hence we may take $\Omega_{\mathcal{H}}=\left\{Q_{n i}: 0 \leq i<\frac{r}{n}\right\}$. For each integer $i \geq 0$, let $\sigma_{i}$ be the restriction of $\sigma_{Q_{i}}$ to $F$. Then $\operatorname{Gal}(F / \mathrm{k})=\left\{\sigma_{i}: 0 \leq i<n\right\}$. For each $0 \leq i<n$, we may take $\beta_{\sigma_{i}}=Q_{i}$, so

$$
f_{\sigma_{i}}(X)=\sum_{h=0}^{\frac{r}{n}-1} X^{\operatorname{deg}_{P}\left(Q_{i} Q_{n h}\right)}=\sum_{h=0}^{\frac{r}{n}-1} X^{\operatorname{deg} Q_{i+n h}} .
$$

Note that $\sigma_{j}^{-1}=\sigma_{n-j}$ for $0 \leq j<n$. Then, for each $1 \leq i, j<n$, we have

$$
f_{i j}(X):=f_{\sigma_{i} \sigma_{j}^{-1}}(X)-f_{\sigma_{i}}(X)=\sum_{h=0}^{\frac{r}{n}-1} X^{\operatorname{deg} Q_{i+n-j+n h}}-X^{\operatorname{deg} Q_{i+n h}} .
$$

Since $F$ has a monic irreducible conductor $P, J_{F}(X)=1$, so we get the following.

Proposition 3.2. Let $F$ be a real Abelian function field with a monic irreducible conductor $P$. For each $1 \leq i, j<n=[F: \mathrm{k}]$, let $f_{i j}(X)$ be as above. Then we have

$$
D_{F}(X)=\left(\frac{f_{i j}(X)}{1-X}\right)_{1 \leq i, j<n}
$$

and $P_{F}(X)=\operatorname{det} D_{F}(X)$. 
TABle 1. $P_{F}(X)$ for the subfield $F$ of $K_{P}$ with $[F: \mathrm{k}]=13$

\begin{tabular}{|c|c|c|}
\hline$P$ & $P_{F}(X)$ & $h(F)$ \\
\hline$T^{3}+2 T+1$ & $\begin{array}{c}1+9 X+42 X^{2}+144 X^{3}+399 X^{4}+900 X^{5}+1691 X^{6} \\
+2700 X^{7}+3591 X^{8}+3888 X^{9}+3402 X^{10}+2187 X^{11}+729 X^{12} \\
\end{array}$ & $3^{9}$ \\
\hline$T^{3}+2 T+2$ & $\begin{array}{c}1+9 X+42 X^{2}+144 X^{3}+399 X^{4}+900 X^{5}+1691 X^{6} \\
+2700 X^{7}+3591 X^{8}+3888 X^{9}+3402 X^{10}+2187 X^{11}+729 X^{12} \\
\end{array}$ & $3^{9}$ \\
\hline$T^{3}+T^{2}+2$ & $\begin{array}{c}1+9 X+42 X^{2}+131 X^{3}+308 X^{4}+601 X^{5}+1067 X^{6} \\
+1803 X^{7}+2772 X^{8}+3537 X^{9}+3402 X^{10}+2187 X^{11}+729 X^{12} \\
\end{array}$ & $53 \cdot 313$ \\
\hline$T^{3}+2 T^{2}+1$ & $\begin{array}{c}1+9 X+42 X^{2}+131 X^{3}+308 X^{4}+601 X^{5}+1067 X^{6} \\
+1803 X^{7}+2772 X^{8}+3537 X^{9}+3402 X^{10}+2187 X^{11}+729 X^{12}\end{array}$ & $53 \cdot 313$ \\
\hline
\end{tabular}

\section{Examples}

Let $F$ be a real Abelian function field with a monic irreducible conductor $P$. In this section, we give some examples of the congruence zeta function $P_{F}(X)$ of $F$ using Proposition 3.2. As the k-isomorphism $T \mapsto T+\alpha$ with $\alpha \in \mathbb{F}_{q}^{*}$ sends a monic irreducible polynomial to another one, it suffices to consider only the monic irreducible polynomials up to these k-isomorphisms.

Example 4.1. Assume $q=3$. There are four monic irreducible polynomials $T^{3}+2 T+1, T^{3}+2 T+2, T^{3}+T^{2}+2$ and $T^{3}+2 T^{2}+1$ of degree 3 up to the above k-isomorphisms. Since $\left[K_{P}: \mathrm{k}\right]=26$, there is only one non-trivial real subfield $F$ of $K_{P}$, which is of degree 13 over k. The table of $P_{F}(X)$ and $h(F)=P_{F}(1)$ for these polynomials are given in Table 1.

Example 4.2. Assume $q=3$. There are six monic irreducible polynomials of degree 4 up to the above k-isomorphisms. We only give the table for $T^{4}+T+2$ in Table 2. Note that for each positive divisor of 40 there is only one real subfield $F$ of $K_{P}$ for the same degree. In the case $[F: \mathrm{k}]=8$, we have the matrix $D_{F}(X)$ as follows;

$$
\left(\begin{array}{ccccccc}
X^{2}+1 & -X^{2}+X & 2 X^{2} & -X & -X^{2} & X^{2}+X & X^{2} \\
-2 X^{2}-X & X+1 & X^{2}+X & 2 X^{2}-X & -X^{2}-X & X & 2 X^{2}+X \\
-X^{2}-X & -3 X^{2} & 2 X^{2}+X+1 & X^{2} & X^{2}-X & 0 & X^{2}+X \\
0 & -2 X^{2} & -X^{2} & 2 X^{2}+1 & 0 & 2 X^{2} & X^{2} \\
-X^{2} & -X^{2}+X & 0 & -X^{2}-X & X^{2}+1 & X^{2}+X & 3 X^{2} \\
-X^{2}-X & -2 X^{2}+X & X^{2}+X & -X & -2 X^{2}-X & 2 X^{2}+X+1 & 2 X^{2}+X \\
X^{2}-X & -2 X^{2} & X & X^{2} & -X^{2}-X & -X^{2} & 3 X^{2}+X+1
\end{array}\right) .
$$

In Table $2, f_{1}(X)$ is given by

$$
\begin{aligned}
& 1+16 X+133 X^{2}+760 X^{3}+3326 X^{4}+11764 X^{5}+\cdots \\
& +17175641679 X^{36}+6198727824 X^{37}+1162261467 X^{38}
\end{aligned}
$$

and $f_{2}(X)$ is given by

$$
\begin{aligned}
& 1+36 X+663 X^{2}+8320 X^{3}+79951 X^{4}+626884 X^{5}+\cdots \\
& +298538229605731251669 X^{76}+48630661836227715204 X^{77} \\
& +4052555153018976267 X^{78}
\end{aligned}
$$

One can see that our data coincide with one in [2]. 
TABle 2. $P_{F}(X)$ for the real subfield $F$ of $K_{P}$ with $P=$ $T^{4}+T+2$

\begin{tabular}{|c|c|c|}
\hline$[F: k]$ & $P_{F}(X)$ & $h(F)$ \\
\hline 2 & $1+3 X^{2}$ & $2^{2}$ \\
\hline 4 & $1+4 X+11 X^{2}+24 X^{3}+33 X^{4}+36 X^{5}+27 X^{6}$ & $2^{3} \cdot 17$ \\
\hline 5 & $1+X-2 X^{2}-5 X^{3}+X^{4}-15 X^{5}-18 X^{6}+27 X^{7}+81 X^{8}$ & 71 \\
\hline 8 & $1+4 X+19 X^{2}+56 X^{3}+153 X^{4}+356 X^{5}+715 X^{6}+1344 X^{7}+2145 X^{8}$ & $2^{4} \cdot 17 \cdot 97$ \\
\hline 10 & $+3204 X^{9}+4131 X^{10}+4536 X^{11}+4617 X^{12}+2916 X^{13}+2187 X^{14}$ & $2^{2} \cdot 71 \cdot 491$ \\
\hline 20 & $1+6 X+18 X^{2}+40 X^{3}+71 X^{4}+70 X^{5}-43 X^{6}-358 X^{7}-1064 X^{8}-2220 X^{9}-3192 X^{10}$ & $2^{3} \cdot 11^{2} \cdot 17$ \\
\hline 40 & $f_{1}(X)$ & $\cdot 71.491 \cdot 541$ \\
\hline & $f_{2}(X)$ & $2^{4} \cdot 11^{2} \cdot 17 \cdot 41 \cdot 71 \cdot 97$ \\
\hline
\end{tabular}

\section{References}

[1] J. Ahn, S. Choi, and H. Jung, Class number formulae in the form of a product of determinants in function fields, J. Aust. Math. Soc. 78 (2005), no. 2, 227-238.

[2] S. Bae, H. Jung, and J. Ahn, Class numbers of some abelian extensions of rational function fields, Math. Comp. 73 (2004), no. 245, 377-386.

[3] S. Bae and P.-L. Kang, Class numbers of cyclotomic function fields, Acta Arith. 102 (2002), no. 3, 251-259.

[4] M. Rosen, A note on the relative class number in function fields, Proc. Amer. Math. Soc. 125 (1997), no. 5, 1299-1303.

[5] D. Shiomi, A determinant formula for congruence zeta functions of maximal real cyclotomic function fields, Acta Arith. 138 (2009), no. 3, 259-268.

JAEHYUN AHN

Department of Mathematics

Chungnam National University

DAEJON 305-764, KoREA

E-mail address: jhahn@cnu.ac.kr

HWANYuP Jung

Department of Mathematics EduCATion

Chungbuk National University

Cheonguu 361-763, Korea

E-mail address: hyjung@chungbuk.ac.kr 